\title{
Influence of a Fungus-Feeding Nematode on Growth and Biocontrol Efficacy of Trichoderma harzianum
}

\author{
Yeoung-Seuk Bae and Guy R. Knudsen
}

Soil Science Division, University of Idaho, Moscow 83844.

Accepted for publication 9 November 2000.

\begin{abstract}
Bae, Y.-S., and Knudsen, G. R. 2001. Influence of a fungus-feeding nematode on growth and biocontrol efficacy of Trichoderma harzianum. Phytopathology 91:301-306.

A fungivorous nematode, Aphelenchoides sp., was isolated from field soil by baiting with mycelium of the biocontrol fungus Trichoderma harzianum ThzID1, and subsequently was maintained on agar cultures of the fungus. Interactions between the nematode and the green fluorescent protein-producing transformant, T. harzianum ThzID1-M3, were investigated in both heat-treated $\left(80^{\circ} \mathrm{C}, 30 \mathrm{~min}\right)$ and untreated field soil. ThzID1-M3 was identified in soil by epifluorescence microscopy. When ThzID1-M3 was added to soil as an alginate pellet formulation, addition

of the nematode (10 per gram of soil) significantly reduced radial growth and recoverable populations of the fungus, and the effect was greater in heat-treated soil than in untreated soil. Addition of ThzID1-M3 to soil pretreated with the nematode (10 per gram of soil) stimulated nematode population growth for approximately 10 to 20 days, whereas nematode populations decreased in the absence of added Trichoderma sp. When sclerotia of Sclerotinia sclerotiorum were added to soil (10 per $200 \mathrm{~g}$ of soil) with ThzID1-M3 (40 pellets per $200 \mathrm{~g}$ of soil), addition of Aphelenchoides sp. (2,000 per $200 \mathrm{~g}$ of soil) reduced the number of sclerotia colonized by ThzID1-M3. These results suggest that fungivorous nematodes may be a significant biotic constraint on activity of biocontrol fungi in the field.
\end{abstract}

Interactions between biocontrol fungi and soil microanimals have largely been neglected in studies relating to the ecology and biocontrol efficacy of fungal agents. Nematodes are the most abundant soil-inhabiting animals. There are more than 80,000 described species, and population densities typically are high in the top $5 \mathrm{~cm}$ of soils, with substantial seasonal differences in agricultural soils $(26,29,30)$. One way that nematologists have characterized the functional role of nematodes in soil communities is according to the nature of their food and method of feeding (25). Identified trophic groups include fungivores, bacterivores, omnivores, predators, and plant parasites $(12,25)$. Fungivorous nematodes have a stylet, and feed on mycelia of many different species of soil fungi, including beneficial fungi as well as plant pathogens $(5,10)$. Numerous species of fungivores have been found in soils (25). The most common genera found in agricultural soils are Aphelenchoides, Aphelenchus, Tylenchus, and Ditylenchus (10,12, 17,30); some species within these genera are plant parasites. For example, Ditylenchus destructor, which is parasitic on carrot, potato, alfalfa, radish, and sweetpotato, also can be cultured on a variety of filamentous fungi (10). Fungivores within this group of nematodes usually are found at lower densities than are bacterivores or plant parasites $(12,27)$. However, if suitable fungi as food are available, populations of fungivores may rapidly increase. Hofman and s'Jacob (17) reported that numbers of the mycophagous nematodes Aphelenchoides sp., Aphelenchus avenae, and unidentified microbivorous nematodes increased several-fold within a few days after being added to flax roots that had been precolonized by Rhizoctonia solani. Some plant ectoparasitic nematodes also may be fungivorous, as the need arises (25).

Isolates of the soil fungus Trichoderma harzianum Rifai have been described as potential biocontrol agents against several soilborne plant pathogens $(15,16,21,28)$. A variety of methods for

Corresponding author: G. R. Knudsen; E-mail address: gknudsen@uidaho.edu

Publication no. P-2001-0108-01R

(c) 2001 The American Phytopathological Society application of biocontrol fungi to soil have been developed, with the goal of enhancing proliferation and establishment of the agent $(11,22,23)$. Incorporation of spores or hyphal biomass into alginate pellets, with or without a nutrient source or other additives, has several advantages, including a relatively long shelf life of the formulated agent and ease of application $(8,11,23)$. When a fungal biocontrol agent is formulated in this manner, the introduced fungus may rapidly grow from the source, form a hyphal network, and potentially contact and colonize target propagules of a soilborne pathogen or other substrata $(3,22)$. Knudsen and Bin (20) reported a hyphal density of 10 to $70 \mathrm{~mm} \mathrm{~mm}^{-3}$ of soil, with a maximum of $30 \mathrm{~mm}$ of hyphal radial extension, within 7 to 14 days for T. harzianum.

Previous research in this laboratory demonstrated effects of several abiotic (temperature, soil moisture, added nutrients) and biotic (potentially antagonistic bacteria) factors on hyphal growth and biocontrol efficacy of $T$. harzianum $(6,8,20,21)$. Some of these experiments were conducted using partially sterilized soil, whereas other experiments in untreated soil were not able to clearly distinguish effects of introduced T. harzianum from possible effects of indigenous Trichoderma spp. Recently, we reported the cotransformation of $T$. harzianum ThzID1 with genes encoding production of green fluorescent protein (GFP), as well as $\beta$-glucuronidase and hygromycin B resistance (3). Expression of GFP allows detection of the introduced agent against a background of indigenous fungi and thus provides a useful tool for evaluating its growth and biocontrol activity in a natural soil community. We hypothesized that one constituent of the soil community, fungivorous nematodes, may adversely affect growth and biocontrol efficacy of the introduced agent. The objectives of this study were to determine (i) whether a fungivorous nematode influences growth and proliferation of ThzID1-M3 in soil, (ii) whether application of formulated ThzID1-M3 to soil stimulates population growth of known fungivorous and other nematodes, and (iii) whether a population of fungivorous nematodes can reduce the ability of ThzID1-M3 to colonize sclerotia of Sclerotinia sclerotiorum in soil. 


\section{MATERIALS AND METHODS}

Nematode isolation and identification. A quantity of Latahco silt loam soil was obtained from the University of Idaho Parker Farm near Moscow. The field had been cropped to potato ('Russet Burbank') for 2 years in succession. Soil analysis (University of Idaho Analytical Services Laboratory) indicated that the soil contained 20\% sand, 20\% clay, and 60\% silt by weight. Soil pH in soil/water (2:1) solution was approximately 5.9. Soil was sieved through a 2-mm mesh and air dried prior to use. T. harzianum ThzID1 was grown in potato dextrose broth shake culture for 3 days at approximately $22^{\circ} \mathrm{C}$. Mycelium was harvested by filtering with four layers of cheesecloth, then washed with sterile distilled water. A sample of the mycelial mat (approximately $5 \mathrm{~g}$ ) was enclosed in nylon mesh (0.1-mm mesh size) and buried in approximately $1 \mathrm{~kg}$ of soil. After 3 days of incubation at approximately $22^{\circ} \mathrm{C}$, the mycelium was harvested and nematodes were extracted from the mycelium with a Baermann funnel. The isolated nematodes were sorted by morphological characteristics using a compound microscope. Based on the morphological characteristics of fungal feeders $(13,25)$, a single male and a single female from each of five morphologically distinct types were transferred onto potato dextrose agar (PDA) plates of T. harzianum and incubated at $25^{\circ} \mathrm{C}$. Nematodes of one morphological type showed rapid growth on ThzID1 cultures. To eliminate possible microbial contaminants, nematodes were subcultured on ThzID1 cultures on Trichoderma sp. selective medium (TSM; 9), then subcultured again on PDA cultures of ThzID1. These nematodes were used in subsequent experiments. For identification, $\approx 100$ nematodes were heat killed $\left(60^{\circ} \mathrm{C}, 10 \mathrm{~min}\right)$ and observed at $\times 1,000$ magnification. Images were captured with a digital video camera and compared with reference drawings and photographs $(13,24)$. Based on the following characteristics, we identified the nematode as a member of the genus Aphelenchoides: shape vermiform with nondistinct cuticular segmentation; relatively short stylet with small distinct knobs; esophagus is a slender precorpus leading to a distinct, well-developed median bulb, and isthmus united imperceptibly with the beginning of the intestine; male tail arcuate with a small pointed terminus, presenting three pairs of caudal papillae, spicules paired and thorn-shaped; female tail tapering and conical with a small terminus.

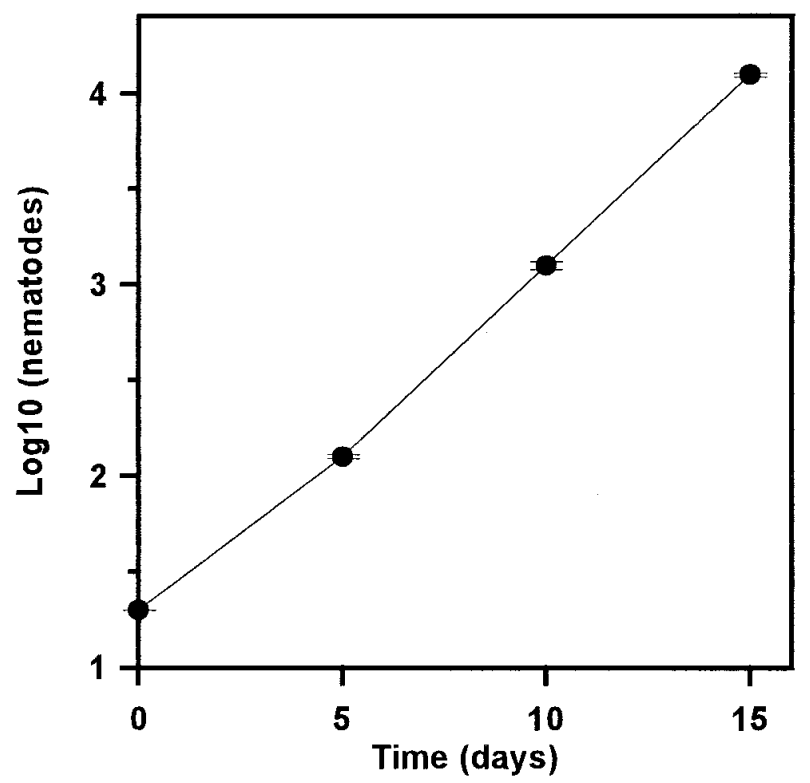

Fig. 1. Numbers of Aphelenchoides sp. in cultures with Trichoderma harzianum grown at $25^{\circ} \mathrm{C}$. Nematodes were extracted $0,5,10$, and 15 days after transfer onto the fungal cultures.
Population growth rate of Aphelenchoides sp. on T. harzianum agar cultures. The population growth rate of Aphelenchoides sp. was investigated in agar culture with $T$. harzianum. The medium used was TSM, which inhibits growth of contaminant bacteria, many fungi, and non-stylet-bearing nematodes (9), but supports limited colony growth of Trichoderma spp. Aphelenchoides sp. adults, 10 males and 10 females, were placed on TSM agar plates on which T. harzianum had been allowed to grow for 3 days. The plates were incubated at $25^{\circ} \mathrm{C}$ in the dark. After 5, 10, and 15 days, plates were destructively sampled by inverting the contents onto four layers of tissue paper (Kimwipes) in a Baermann funnel filled with sterile distilled water. Nematodes were extracted over a 6-day period, and total counts were obtained with a dissecting microscope. There were three replicates of each treatment for each sample time and the experiment was conducted twice.

Effect of Aphelenchoides sp. on radial growth of T. harzianum in soil. Latahco silt loam soil was either air dried as above ("untreated soil"), or air dried and then heat treated ("heat-treated soil") by wetting with sterile water to a soil moisture potential of $-100 \mathrm{kPa}$, then placing $\approx 1-\mathrm{kg}$ samples at $80^{\circ} \mathrm{C}$ for $30 \mathrm{~min}$. The latter treatment effectively eliminated populations of indigenous nematodes and Trichoderma spp. as well as most indigenous fungi and bacteria (data not shown). Both untreated and heat-treated soils were adjusted a soil moisture content of $-50 \mathrm{kPa}$ by adding either sterile water or water containing Aphelenchoides sp. from ThzID1 cultures $(1,000$ nematodes per $100 \mathrm{~g}$ of dry soil, air-dried basis).

The strain of T. harzianum used in this experiment was ThzID1M3, which was transformed with genes encoding hygromycin B resistance, $\beta$-glucuronidase (GUS), and GFP (3). Alginate pellets containing hyphal biomass of ThzID1-M3 plus wheat bran and polyethylene glycol 8000 were formulated as described previously $(20,22)$. Pellets were approximately $1.8 \mathrm{~mm}$ in diameter. Colony growth of ThzID1-M3 was measured by previously described methods (3). Glass petri dishes (15-cm diameter) were half filled with soil (treatments as described above and a glass microscope slide precoated with $1.8 \%$ water agar was placed on the soil surface. A single ThzID1-M3 pellet was attached to the slide surface with cyanoacrylate glue. Dishes then were filled with additional soil, placed in a covered plastic container lined with moist paper towels, and incubated at $25^{\circ} \mathrm{C}$ for $3,5,7$, or 14 days. At each sample time, glass slides were removed and hyphal growth on the glass surface was examined using epifluorescence microscopy. Colony radii were quantified by measuring captured video images at magnifications of $\times 250$ or $\times 400$. There were four replicates per treatment, and the experiment was conducted twice.

Effect of Aphelenchoides sp. on recoverable numbers of Trichoderma propagules, and nematode population dynamics in soil. Untreated or heat-treated soil as described above was mixed with sterile distilled water or nematode suspensions of Aphelenchoides sp. to provide a matric potential of $-50 \mathrm{kPa}$ and nematode population levels of 0 or 500 per $50 \mathrm{~g}$ of soil. Glass containers $(250-\mathrm{ml})$ were filled with $50 \mathrm{~g}$ of either untreated or heat-treated soil, with or without nematodes. Then, either 0 or 20 ThzID1-M3 pellets were added (i.e., a 2-by-2-by-2 factorial design). The containers were covered with aluminum foil and incubated at $25^{\circ} \mathrm{C}$ in the dark for $5,10,20$, or 30 days. There were three replicates per treatment for each sample time, and the experiment was conducted twice. At each sample time, recoverable populations of ThzID1-M3 and total recoverable Trichoderma spp. were determined as follows. The soil in the glass container was thoroughly mixed by shaking, after which a $1-\mathrm{g}$ sample was removed and suspended in $9 \mathrm{ml}$ of sterile distilled water. Serial dilutions were made and aliquots $(100-\mu \mathrm{l})$ were spread on duplicate plates of TSM amended with hygromycin B at $100 \mu \mathrm{g} / \mathrm{ml}$ for enumeration of ThzID1-M3, and with unamended TSM for total Trichoderma spp. Plates were incubated at $25^{\circ} \mathrm{C}$, and colonies were counted after 7 to 10 days. Trichoderma spp. were identified 
to genus by colony color and conidiophore morphology. To enumerate nematode populations, a 20 -g soil sample was obtained from each container and placed on four layers of tissue paper in a Baermann funnel filled with sterile distilled water. Extracts were collected over a 6-day period and counts of all nematodes present (including any indigenous nematodes in untreated soil) were made using a dissecting microscope.

Effect of Aphelenchoides sp. on colonization of sclerotia of $S$. sclerotiorum by ThzID1-M3. Mycelial disks of S. sclerotiorum grown on PDA for 7 days were transferred to sterilized sliced carrots in 2-liter Erlenmeyer flasks. After 6 to 8 weeks at $16^{\circ} \mathrm{C}$, sclerotia were harvested, rinsed with sterile distilled water, and air dried for 2 to 3 days. Sclerotia were attached to plastic toothpicks with cyanoacrylate glue and allowed to dry overnight. All sclerotia were then surface disinfested (10\% ethanol, $10 \%$ bleach in water) for $1 \mathrm{~min}$ and stored at $4^{\circ} \mathrm{C}$ before use.

A 200-g amount of either untreated or heat-treated soil was added to glass screwcap jars (9-cm diameter by $9-\mathrm{cm}$ depth). Forty pellets of ThzID1-M3 were added to each glass jar and thoroughly mixed with soil; then, 10 sclerotia were randomly placed in the soil at a depth of approximately $2 \mathrm{~cm}$. Soil was adjusted to a moisture content of $-50 \mathrm{kPa}$ with sterile distilled water or nematode suspension (to reach 2,000 nematodes per jar). The jars were closed and incubated at $25^{\circ} \mathrm{C}$ for 14 days in the dark. All jars contained sclerotia and ThzID1 pellets, so that the experimental design was a factorial experiment with two levels of soil treatment (untreated and heat-treated) and two levels of nematode infestation ( 0 and 2,000 nematodes per jar). There were five replicates per treatment, and the experiment was conducted twice. After 14 days, sclerotia were recovered, surface sterilized, and placed on PDA containing streptomycin at $50 \mu \mathrm{g} / \mathrm{ml}$ to avoid bacterial contamination. After incubation at $25^{\circ} \mathrm{C}$ for 7 days, sclerotia were observed to determine whether S. sclerotiorum, Trichoderma spp., or other fungi were growing from them. When hyphae of Trichoderma spp. were observed, they were examined for GFP activity using epifluorescence microscopy and proportions of sclerotia colonized by indigenous (non-GFP) Trichoderma spp. versus ThzID1-M3 were recorded.

Statistical analysis. Factorial analyses of variance (ANOVA) of all data except growth data of Aphelenchoides sp. were performed using Statistical Analysis System software (SAS Institute Inc., Cary, NC). Statistical analyses and inferences were based on $\log$ transformations for all population data and based on arcsin transformation for proportion data. Each experiment was conducted twice; treatment by experiment interactions was not significant; therefore, results from both sets of experiments were pooled. Treatment effects were considered to be statistically significant at the $\alpha=0.05$ level. Means and standard errors of the means were calculated for the number of colony forming units (log transformed) of Trichoderma spp. population and for the number of total nematodes observed at each sampling time.

\section{RESULTS}

Population growth rate of Aphelenchoides sp. on T. harzianum agar cultures. Growth of Aphelenchoides sp. on T. harzianum cultured on TSM was approximately exponential (Fig. 1), with an intrinsic population growth rate of $\approx 0.43$ nematodes nematode ${ }^{-1}$ day $^{-1}$. Ratios of male/female and adult/juvenile were determined for a randomly selected sample after 15 days. The male/female ratio was approximately $1: 1.5$; the adult/juvenile ratio was approximately $1: 1.4$.

Effect of Aphelenchoides sp. on radial growth of T. harzianum in soil. Data were analyzed over all sample times. Each of the main effects (soil, nematode, sample time) had a statistically significant effect on radial growth of ThzID1-M3 $\left(P_{\text {soil }}=0.001\right.$, $P_{\text {nematode }}=0.002$, and $\left.P_{\text {sample time }}=0.001\right)$. Colony radius was less in untreated soil compared to heat-treated soil, was less when nematodes were present, and generally increased over time in all treatments. The soil-sample time interaction was significant $(P=$ 0.01 ); thus, soil effects on radial growth were greatest at the 14-day sample time. However, mean colony radius in the heat-treated soil was higher than that in untreated soil at every sample time. The soil-nematode interaction was not significant $(P=0.21)$. In heattreated soil, mean colony radius at day 14 was $9.8 \mathrm{~mm}$ in the absence of Aphelenchoides sp. compared with $6.8 \mathrm{~mm}$ with the nematode present (Fig. 2). In untreated soil, mean colony radius at day 14 averaged $5.2 \mathrm{~mm}$ in the absence of Aphelenchoides sp. compared with $4.3 \mathrm{~mm}$ with the nematode present (Fig. 2).

Effect of Aphelenchoides sp. on population growth of Trichoderma and nematode population dynamics in soil. Mean recoverable numbers (log transformed) of ThzID1-M3 are shown in Figure 3. Over all sample times, soil treatment and presence of nematodes were statistically significant in their effect on numbers of recoverable propagules of ThzID1-M3 $\left(P_{\text {soil }}=0.0001, P_{\text {nematode }}=\right.$ 0.0001). Recoverable numbers of ThzID1-M3 were higher in heattreated than in untreated soil at all sampling times, regardless of whether nematodes were present or absent (Fig. 3), and addition of the nematode significantly reduced recoverable populations of ThzID1-M3 (Fig. 3). Interactions between factors were not significant $(P>0.05)$.

Increased population densities of total recoverable Trichoderma spp. in both soils resulted from the addition of ThzID1-M3, with higher population levels attained in heat-treated soil $(P=0.001$, Fig. 4). In untreated soil, mean numbers of total Trichoderma spp. were approximately 10- to 15-fold higher when ThzID1-M3 was added (Fig. 4). Treatment with Aphelenchoides sp. in both soils significantly reduced total populations of Trichoderma spp. $(P=$ 0.001 ; Fig. 4). However, this effect was confounded by a significant soil-nematode interaction $(P=0.01)$, because low levels of Trichoderma spp. apparently were introduced as contaminants along with the nematodes (which had been reared on Trichoderma spp. cultures) (Fig. 4).

Addition of ThzID1-M3 stimulated populations of nematodes in untreated soil, regardless of whether Aphelenchoides nematodes

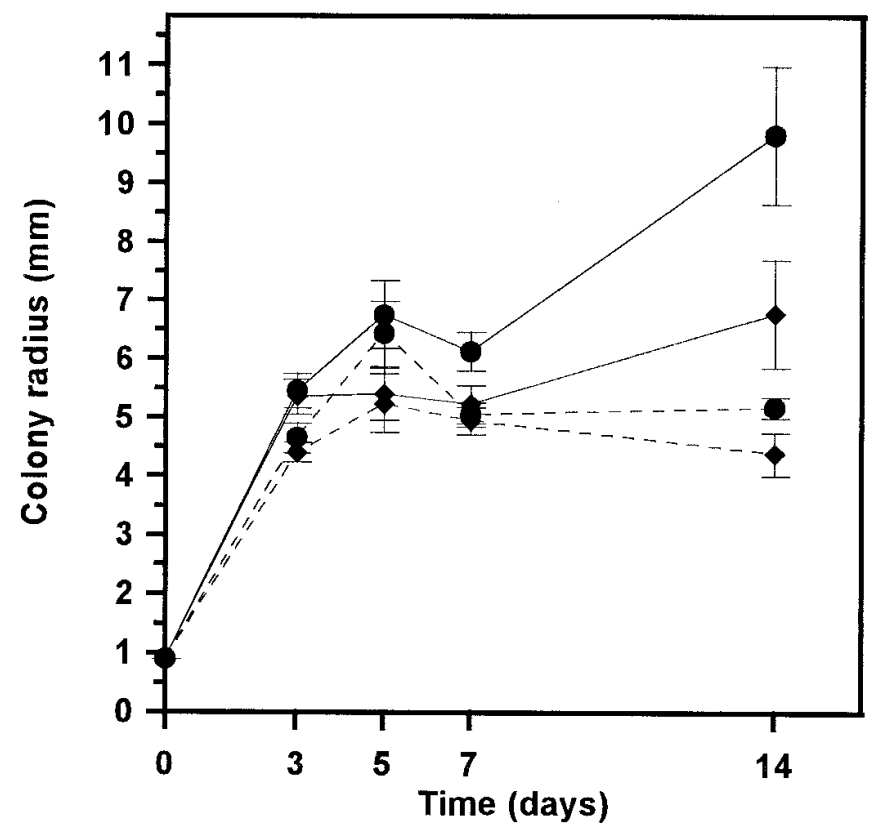

Fig. 2. Effect of Aphelenchoides sp. on radial growth of Trichoderma harzianum isolate ThzID1-M3 originating from alginate pellets in heattreated (solid line) or untreated (dotted line) soil at 0, 3, 5, 7, and 14 days. Aphelenchoides sp. was applied to soil at initial levels of $0(\bullet)$ or $10(\diamond)$ per gram of dry soil. Radial growth was measured at a magnification of $\times 250$ or $\times 400$ under epifluorescence microscopy. Vertical bars represent \pm 1 standard error of the mean. 
were added (Fig. 5). Indigenous nematodes initially were at undetectable levels and did not increase when ThzID1-M3 was not added (Fig. 5). When individuals of Aphelenchoides sp. were added to untreated soil, numbers of total recoverable nematodes decreased over time if ThzID1-M3 was not also added (Fig. 5). Results were similar for the heat-treated soil, except that indigenous nematodes apparently were eliminated by the heat treatment (Fig. 5).

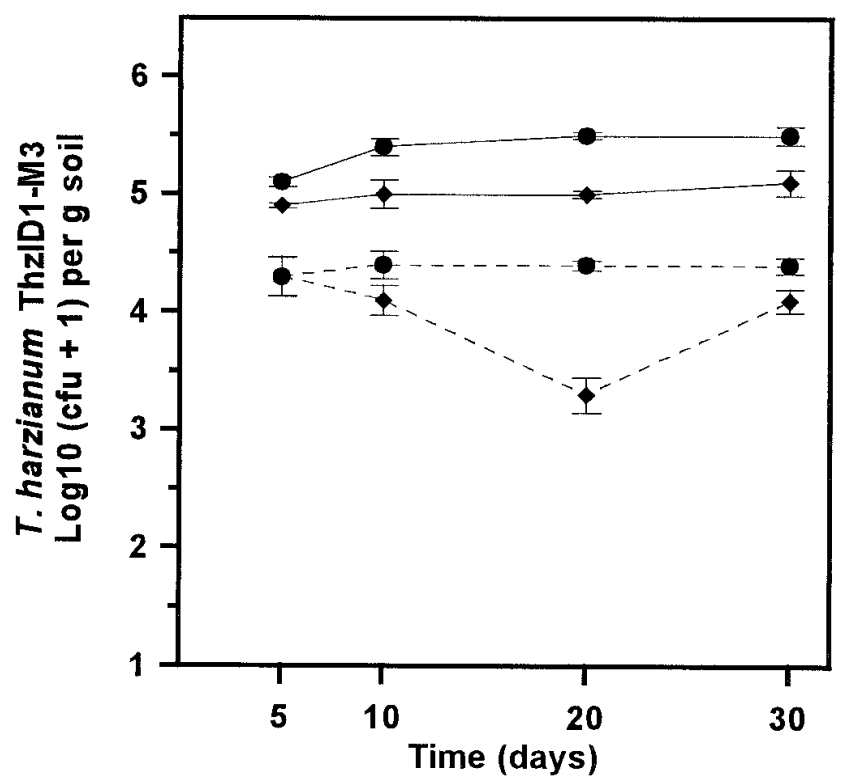

Fig. 3. Effect of Aphelenchoides sp. on populations of Trichoderma harzianum isolate ThzID1-M3 in heat-treated (solid line) or untreated (dotted line) soil at 5, 10, 20, and 30 days. Aphelenchoides sp. was applied to soil at initial levels of $0(\bullet)$ or $10(\downarrow)$ per gram of dry soil, with 20 alginate pellets (per $50 \mathrm{~g}$ of dry soil) of ThzID1-M3. Vertical bars represent \pm 1 standard error of the mean.
Effect of Aphelenchoides sp. on colonization of sclerotia by ThzID1-M3. Mean proportions of sclerotia colonized by ThzID1M3 are shown in Figure 6. Both main effects (soil, $P=0.0002$; nematode, $P=0.004$ ) had significant effects on colonization by ThzID1-M3, although proportions of sclerotia colonized by indigenous Trichoderma spp. (i.e., Trichoderma spp. other than ThzID1-M3) did not differ significantly among treatments $(P=$ $0.25)$. Numbers of sclerotia colonized by ThzID1-M3 were higher overall in heat-treated soil than in untreated soil, and addition of Aphelenchoides sp. resulted in lower numbers, in both soils, of sclerotia colonized by ThzID1-M3 compared with untreated controls. The soil-nematode interaction was not significant.

\section{DISCUSSION}

The effectiveness of biocontrol fungi against soilborne plant diseases may depend in part on their ability to proliferate through the three-dimensional soil space via hyphal growth originating from the introduced source $(21,22)$. There are many biotic and abiotic factors, including temperature, soil moisture, nutrients, and microorganisms or microanimals, that may affect the growth and efficacy of biocontrol fungi in soil $(6,8,18,20)$. We demonstrate here that a fungal-feeding nematode potentially can reduce the growth and biocontrol efficacy of T. harzianum in soil.

Fungivorous nematodes such as Aphelenchoides sp. and Aphelenchus avenae are associated with saprophytic, pathogenic, and mycorrhizal fungi in soil $(5,10,19)$. Several fungus-feeding nematodes can be cultured on media with an appropriate fungus. For example, the potato rot nematode Ditylenchus destructor fed and reproduced on 64 out of 115 soil-inhabiting fungi representing 40 genera and 8 orders (10). In our study, we isolated Aphelenchoides $\mathrm{sp}$. by baiting soil from a field previously cropped to potato with mycelium of $T$. harzianum. Aphelenchoides spp. are reported to be common and abundant in potato fields (17). Although our method may have selected for nematodes that were able to aggressively feed on mycelium of $T$. harzianum, we did not examine other soil fungi for their potential food value for this nematode. Aphelenchoides sp. successfully reproduced on PDA or
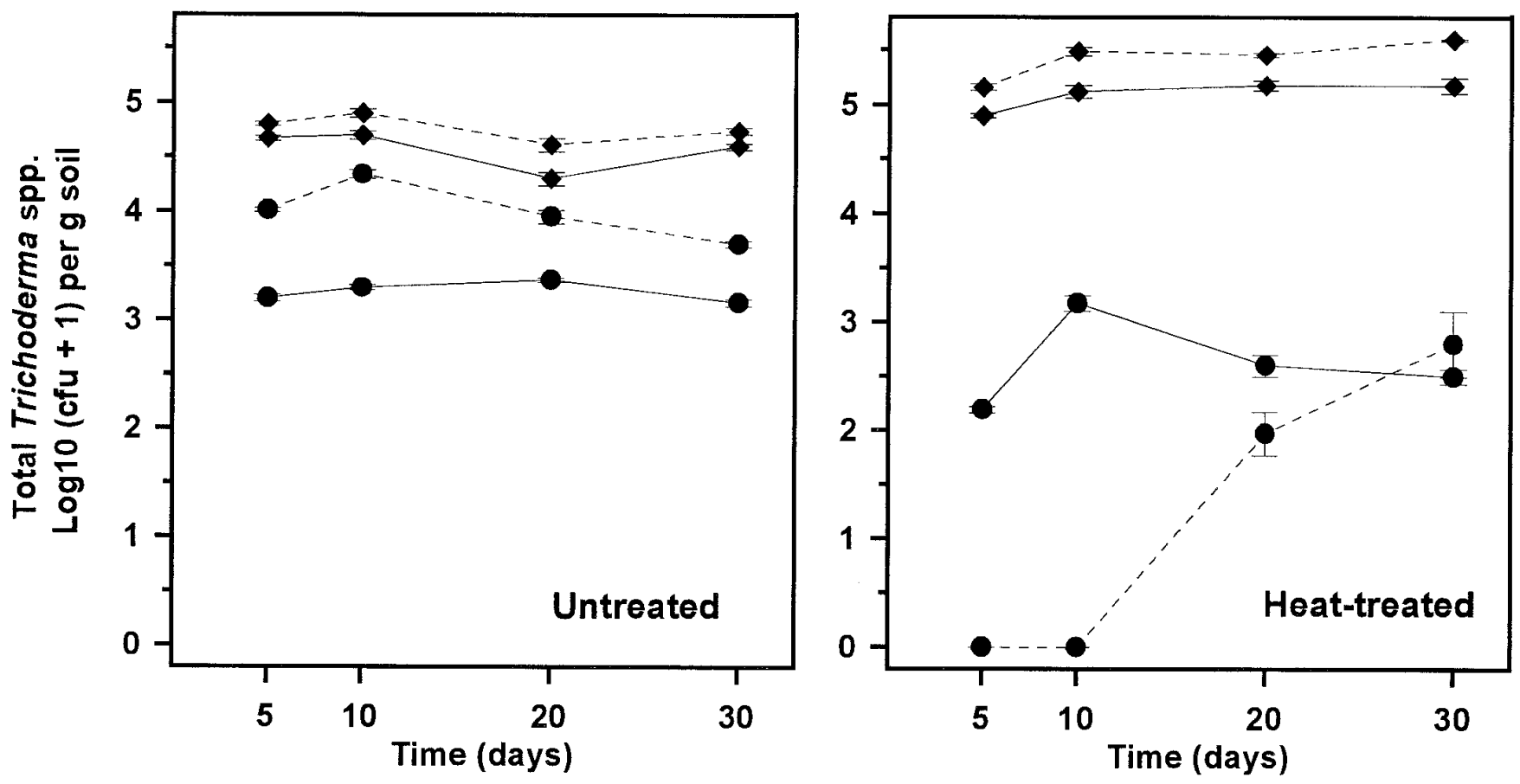

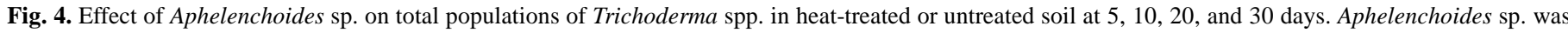

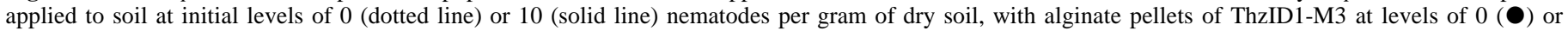
$20(\diamond)$ pellets per $50 \mathrm{~g}$ of dry soil. Vertical bars represent \pm 1 standard error of the mean. 
TSM cultures of T. harzianum, and exhibited exponential growth on TSM with T. harzianum isolate ThzID1 as a food source. Within 10 days after inoculating fungal cultures with nematodes, there were no aerial mycelia observable and, after approximately 10 days, the amount of visible mycelium in culture plates appeared to be greatly diminished.

By using heat-treated soil in some experiments, we tried to eliminate the possibility of direct effects of indigenous nematodes or fungi either on the growth and biocontrol efficacy of ThzID1M3 or on the growth of Aphelenchoides sp. Addition of the nematode significantly reduced radial growth and recoverable populations of ThzID1-M3 added to soil as an alginate pellet formulation both in heat-treated and untreated soil. The addition of ThzID1-M3 to soil pretreated with the nematode stimulated nematode population growth for $\approx 10$ to 20 days, whereas nematode populations decreased in the absence of ThzID1-M3 in both soils. When Aphelenchoides sp. and ThzID1-M3 were added to soil together, total nematode populations were lower in untreated soil than in heat-treated soil. It is not clear whether this result was caused by the presence of other microorganisms or by lower growth and proliferation of the food source (ThzID1-M3) in untreated soil. Untreated soil may contain nematophagous fungi, bacteria, and predacious nematodes $(4,12)$. Field soils may harbor many other fungivorous nematodes, including $A$. avenea, which may occur at high densities $(5,7)$. Cultivation of crops influences the diversity and population of nematodes in the field $(12,30)$. Some fungi, such as Aspergillus and Penicillium spp., also produce nematicidal substrates that can cause direct lethal effects in soil (1). Addition of ThzID1-M3 pellets significantly stimulated the population growth of indigenous nematodes. These might be fungivores, bacterivores, or other nematodes, but quite likely they mainly are fungivores stimulated by ThzID1-M3 addition. In our experiments, we observed many nematodes associated with Trichoderma hyphae around pellets and presumably feeding, and numerous nematode eggs were seen to be adhering to hyphae. These results suggest that populations of fungivores may rapidly increase following addition of biocontrol fungi in the field, consequently inhibiting the growth and proliferation of the added fungi. Compared with overall results from heat-treated soil, the effects of Aphelenchoides sp. on ThzID1-M3 were reduced in untreated soil.
These results support the contention that heat-treated soil has reduced populations of competitive or antagonistic microflora that may influence the growth of introduced biocontrol agents as well as that of the nematodes.

Colonization of sclerotia of S. sclerotiorum by ThzID1-M3 was significantly reduced by the addition of the nematode in both soils. Some soil insects may stimulate colonization of sclerotia by Trichoderma spp. by damaging sclerotia in soil $(2,14)$. However, our results suggest that nematodes did not stimulate colonization

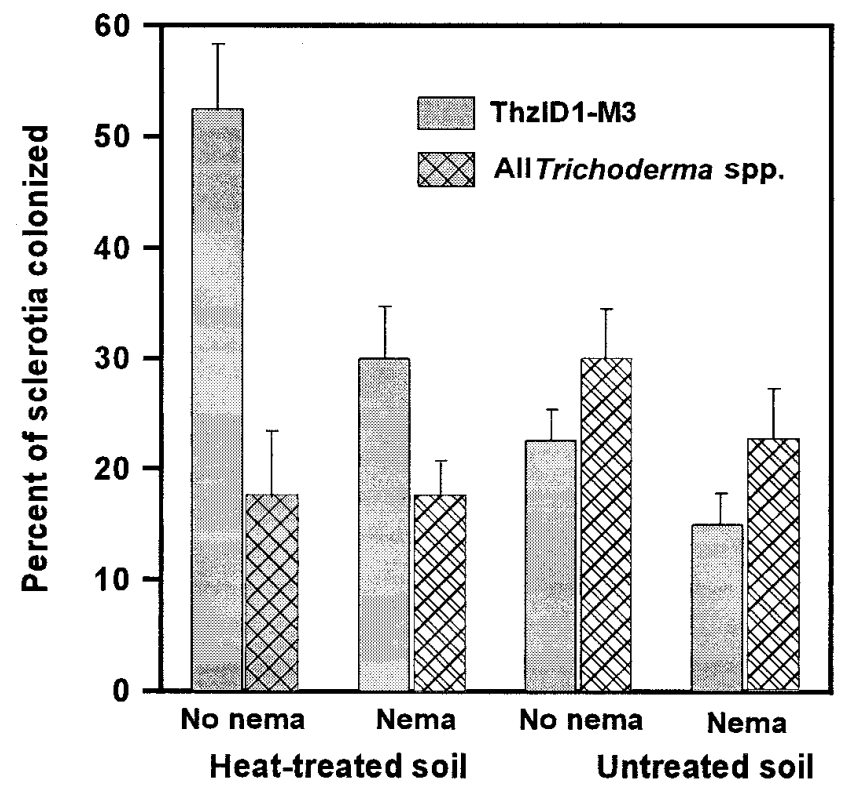

Fig. 6. Effect of Aphelenchoides sp. on colonization of sclerotia of Sclerotinia sclerotinia by Trichoderma harzianum (ThzID1-M3 versus total Trichoderma spp.) in heat-treated or untreated soil. Aphelenchoides sp. was applied to soil at initial levels of 0 or 10 nematodes per gram of dry soil, along with two alginate pellets of ThzID1-M3 per $10 \mathrm{~g}$ of soil. Ten sclerotia were randomly distributed in $200 \mathrm{~g}$ of soil, and colonization was assessed after 14 days. Vertical bars represent \pm 1 standard error of the mean.
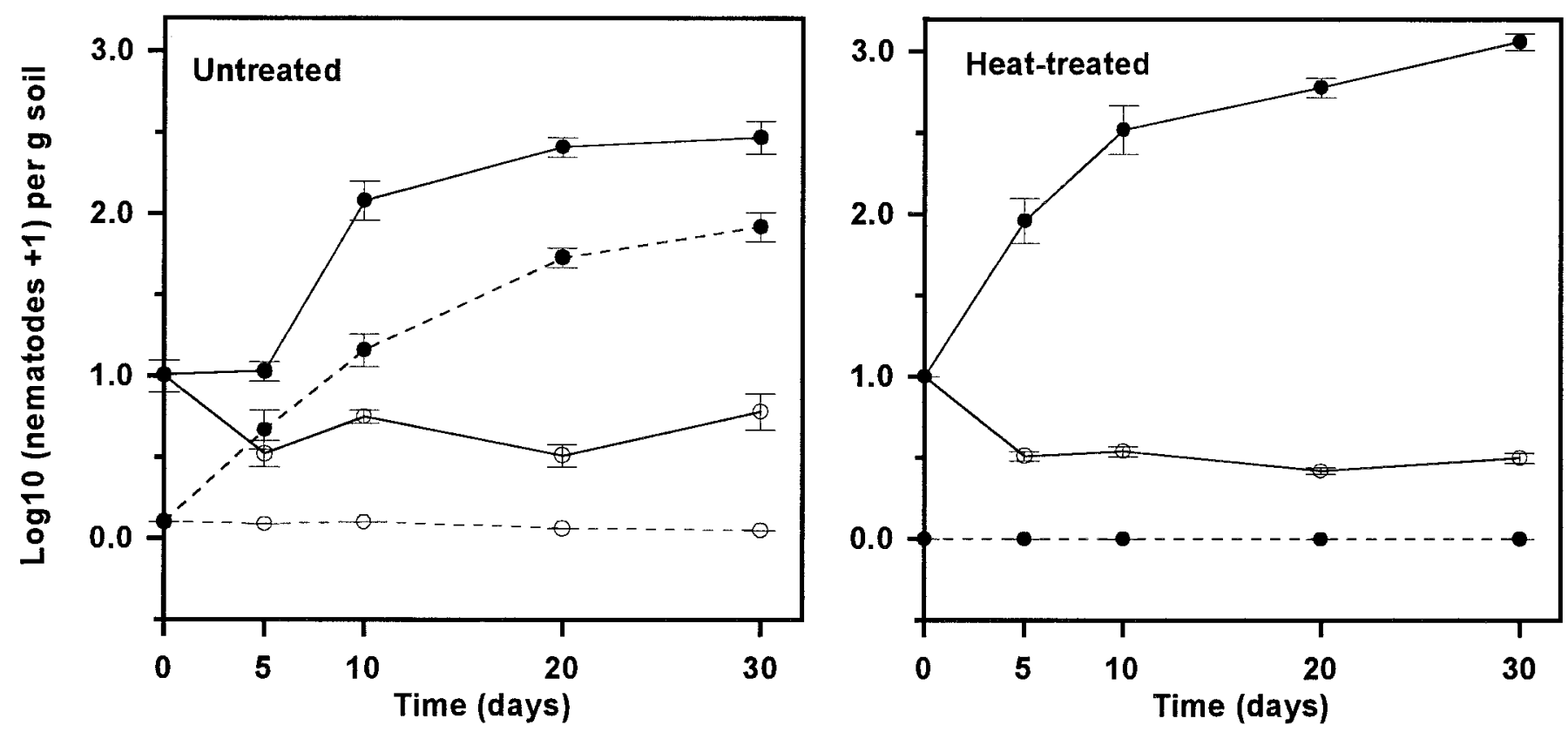

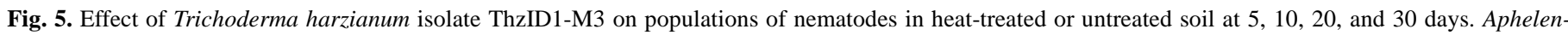

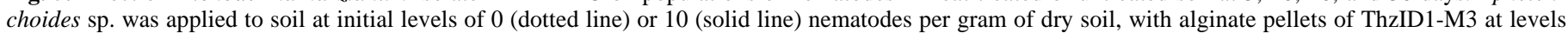
of $0(\bullet)$ or $20(\diamond)$ pellets per $50 \mathrm{~g}$ of dry soil. Vertical bars represent \pm 1 standard error of the mean. 
of sclerotia by Trichoderma spp. in soil. Fungivorous nematodes migrate to and reproduce at locations in the soil where food is available in large quantities (17). The introduced Trichoderma sp. formulated in pellets forms a high-density hyphal net in soil $(3,20)$, and this transient growth of Trichoderma spp. may attract nematodes and serve as an appropriate food source. In conclusion, the results of our study suggest that fungivorous nematodes may, in some circumstances, be significant constraints on the efficacy of biocontrol fungi, so that further investigation of interactions between biocontrol agents and indigenous nematodes under field conditions is warranted.

\section{LITERATURE CITED}

1. Ali, H. H. A. 1990. Nematicidal action of fungal culture filtrates. Jpn. J. Nematol. 20:1-7.

2. Anas, O., and Reeleder, R. D. 1987. Consumption of sclerotia of Sclerotinia sclerotiorum by larvae of Bradysia coprophila: Influence of soil factors and interactions between larvae and Trichoderma viride. Soil Biol. Biochem. 20:619-624.

3. Bae, Y.-S., and Knudsen, G. K. 2000. Cotransformation of Trichoderma harzianum with $\beta$-glucuronidase and green fluorescent protein genes provides a useful tool for monitoring fungal growth and activity in natural soils. Appl. Environ. Microbiol. 66:810-815.

4. Barker, K. R., and Koenning, S. R. 1998. Developing sustainable system for nematode management. Annu. Rev. Phytopathol. 36:165-205.

5. Barnes, G. L., Russell, C. C., Foster, W. D., and McNew, R. W. 1981. Aphelenchus avenae, a potential biological control agent for root rot fungi. Plant Dis. 65:423-424.

6. Bin, L., Knudsen, G. R., and Eschen, D. J. 1991. Influence of an antagonistic strain of Pseudomonas fluorescens on growth and ability of Trichoderma harzianum to colonize sclerotia of Sclerotinia sclerotiorum in soil. Phytopathology 81:994-1000.

7. Bollen, G. L., Middelkoop, J., and Hofman, T. W. 1991. Effects of soil fauna on infection of potato sprouts by Rhizoctonia solani. Pages 27-34 in: Biotic Interactions and Soilborne Diseases. Proc. First Conf. Eur. Foundation Plant Pathol. A. B. R. Beemster, G. J. Bollen, M. Gerlagh, M. A. Ruissen, B. Schippers, and A. Tempel, eds. Elsevier Science Publishing Co., New York.

8. Dandurand, L. M., and Knudsen, G. R. 1993. Influence of Pseudomonas fluorescens on hyphal growth and biocontrol activity of Trichoderma harzianum in the spermosphere and rhizosphere of pea. Phytopathology 83:265-270.

9. Elad, Y., Chet, I., and Henis, Y. 1981. A selective medium for improving quantitative isolation of Trichoderma spp. from soil. Phytoparasitica 9:245-254.

10. Faulkner, L. R., and Darling, H. M. 1961. Pathological history, hosts, and culture of the potato rot nematode. Phytopathology 51:778-786.

11. Fravel, D. R., Marois, J. J., Lumsden, R. D., and Connick, W. J., Jr. 1985. Encapsulation of potential biocontrol agents in an alginate-clay matrix. Phytopathology 75:774-777.

12. Freckman, D. W., and Caswell, E. P. 1985. The ecology of nematodes in agroecosystems. Annu. Rev. Phytopathol. 23:275-296.

13. Goodey, T. 1963. Soil and Freshwater Nematodes. 2nd ed. Methuen, London.

14. Gracia-Garza, J. A., Reeleder, R. D., and Paulitz, T. C. 1997. Degradation of sclerotia of Sclerotinia sclerotiorum by fungus gnats (Bradysia coprophila) and the biocontrol fungi Trichoderma spp. Soil Biol. Biochem. 29:123-129.

15. Hadar, Y., Harman, G. E., and Taylor, A. G. 1984. Evaluation of Trichoderma koningii and T. harzianum from New York soils for biological control of seed rot caused by Pythium spp. Phytopathology 74:106-110.

16. Harman, G. E., Chet, I., and Baker, R. 1980. Trichoderma harzianum effects on seed and seedling disease induced in radish and pea by Pythium spp. or Rhizoctonia solani. Phytopathology 70:1167-1172.

17. Hofman, T. W., and s'Jacob, J. J. 1989. Distribution and dynamics of mycophagous and microbivorous nematodes in potato fields and their relationship to some food sources. Ann. Appl. Biol. 115:219-298.

18. Hubbard, J. P., Harman, G. E., and Hadar, Y. 1983. Effect of soilborne Pseudomonas spp. on the biological control agent, Trichoderma hamatum, on pea seeds. Phytopathology 73:655-659.

19. Hussey, R. S., and Roncadori, R. W. 1982. Influence of Aphelenchus avenae on vesicular-arbuscular endomycorrhizal growth response in cotton. J. Nematol. 13:48-52.

20. Knudsen, G. R., and Bin, L. 1990. Effects of temperature, soil moisture, and wheat bran on growth of Trichoderma harzianum from alginate pellets. Phytopathology 80:724-727.

21. Knudsen, G. R., Eschen, D. J., Dandurand, L. M., and Bin, L. 1991. Potential for biocontrol of Sclerotinia sclerotiorum through colonization of sclerotia by Trichoderma harzianum. Plant Dis. 75:466-470.

22. Knudsen, G. R., Eschen, D. J., Dandurand, L. M., and Wang, Z. G. 1991. Method to enhance growth and sporulation of pelletized biocontrol fungi. Appl. Environ. Microbiol. 57:2864-2869.

23. Lewis, J. A., and Papavizas, G. C. 1987. Applications of Trichoderma and Gliocladium in alginate pellets for control of Rhizoctonia dampingoff. Plant Pathol. 36:438-446.

24. Mai, W. F., and Mullin, P. G. 1996. Plant-Parasitic Nematodes: A Pictorial Key to Genera. 5th ed. Cornell University Press, Ithaca, NY.

25. Nicholas, W. L. 1984. The Biology of Free-Living Nematodes. 2nd ed. Clarendon, Oxford.

26. Paul, E. A., and Clark, F. E. 1996. Components of the soil biota. Pages 69-107 in: Soil Microbiology and Biochemistry. 2nd ed. Academic Press, San Diego, CA.

27. Sohlenius, B. 1980. Abundance, biomass and contribution to energy flow by soil nematodes in terrestrial ecosystems. Oikos 34:186-194.

28. Wells, H. D., Bell, D. K., and Jaworski, C. A. 1972. Efficacy of Trichoderma harzianum as a biocontrol for Sclerotium rolfsii. Phytopathology 62:442-447.

29. Winslow, R. D. 1960. Some aspects of the ecology of free-living and plant-parasitic nematodes. Pages 341-415 in: Nematology. Fundamentals and Recent Advances with Emphasis on Plant Parasitic and Soil Forms. J. N. Sasser and W. R. Jenkins, eds. The University of North Carolina Press, Chapel Hill.

30. Yeates, G. W., Wardle, D. A., and Watson, R. N. 1999. Responses of soil nematode populations, community structure, diversity and temporal variability to agricultural intensification over a seven-year period. Soil Biol. Biochem. 31:1721-1733. 